\title{
The Multiple Outputs of Energetic Pulsars
}

\author{
Roger W. Romani \\ KIPAC/Dept. of Physics, Stanford University, \\ MC 4060, Stanford, CA, USA, 94305 \\ email: rwr@astro.stanford.edu
}

\begin{abstract}
This talk commented on our progress in understanding high $\dot{E}$ pulsar output, with the photon power dominated by $\mathrm{GeV}$ radiation and the total power dominated by the $e^{ \pm} / B$ wind. We are increasingly appreciating the anisotropy in these outflows, with high energy pulsar beaming probed by the distribution of $\gamma$-ray pulse profiles and wind anisotropy mapped by synchrotron images of PWNe. Possible hemispheric asymmetry and the prospects for additional probes of pulsar spindown, particularly from compact binaries in the black widow class, are briefly mentioned.
\end{abstract}

Keywords. pulsars: general, binaries, ISM: supernova remnants

\section{Introduction}

We have long known that energetic pulsars only emit $10^{-6}-10^{-9} \dot{E}$ in the radio. In the past decade Fermi has abundantly confirmed the hints of earlier missions that for energetic $\dot{E}>10^{34} \mathrm{erg} \mathrm{s}^{-1}$ pulsars the dominant photon power is in the $\mathrm{GeV} \gamma$-rays. The exceptions are the very young Crab-like pulsars, for which the $\mathrm{GeV}$ emission may be overshadowed by a few-MeV peak. The variety of Fermi detections, however, have made several additional points clear: GeV domination is also true for the energetic MSP and this radiation is beamed preferentially to the spin equator.

For the young pulsars this pattern points to an outer magnetosphere origin. Phenomenology employing vacuum gaps identified the emission as arising in caustic-producing sheets associated with the last closed field lines approaching the light cylinder (Watters et al. 2009). More recently numerical simulation of resistive magnetospheres points to equatorial current sheets at and just beyond the light cylinder (Philippov \& Spitkovsky 2017; Kalapathorakos et al. 2017). In either case the emission can be traced to a very wide, pole-linked structure near or beyond $R_{L C}$. The dominant radiation mechanism is still under debate with pair-starved models invoking large $\gamma \sim 10^{7}$ limited by $\mathrm{GeV}$ curvature radiation reaction, while the current sheet picture focuses on $\gamma \sim 10^{4}$ particles producing $\mathrm{GeV}$ synchrotron radiation and less capable of pair production. But in either case the GeV flux (and beaming) traces the dominant energy loss mechanism (and spatial distribution) of the radiation reaction-limited particles.

But, as early Crab studies hinted, even the high energy output is $<10^{-2} \dot{E}$ reaching $>10 \%$ of the spindown power only for the 'electrodynamically challenged' pulsars below $\sim 10^{34} \mathrm{erg} \mathrm{s}^{-1}$. This is a natural consequence of gap models and may well arise in current sheet models as well. The bulk of the spindown power - the $e^{ \pm} / B$ pulsar wind that completes the pulsar circuit - is initially dark. This wind at launch is cold, so we see subluminous central zones in pulsar wind nebulae (PWNe). Remarkably, while it is is widely believed that the wind is increasingly particle dominated at large radii, the conversion of Poynting flux to particles keeps the wind cold and disipationless until static or ram pressure with an internal medium forces a termination shock. There synchrotron emission provides a (often resolved) map of the wind and its dissipation. 
This general picture seems quite healthy, but as we seek for specifics about the dominant particle populations in the magnetosphere and wind zone we have to consider a range of newly observed phenomena. These provide the phenomenological hints that will, it is hoped, allow us to connect back to first principles models. I continue by summarizing some recent surprises in this arena that suggest we have much yet to learn about the dominant pulsar output. The list is perforce idiosyncratic, focusing on what interests me at the moment.

\section{Some New Phenomena}

The Fermi all sky survey provides many benefits including a uniform census of pulsar $\gamma$-ray activity. The second pulsar catalog (2PC, Abdo et al. 2013) already contained 117 pulsars (42 young radio loud, 35 young radio quiet and $40 \mathrm{MSP}$ ). The next catalog should nearly double this number with $\sim 210$ publicly announced $\gamma$-ray PSR at this writing. This includes the first (and so far only) $\gamma$-quiet MSP (Clark et al. these proceedings). With so many detections some conclusions are very robust: for the young pulsars the radio beams are not co-located with the GeV emission, and pulse phenomenology (e.g. Watters et al. 2009; Pierbattista et al. 2015) assigns the radio beams to the magnetic pole (as usual) and the $\gamma$-ray beams to the spin equator. This leads to a radio quiet fraction $>1 / 2$ in the young pulsar population. For the MSP, in contrast, the $\gamma$-ray beams must be very wide, nearly always sweeping out the same sky as covered (generally for a much smaller portion of the duty cycle) by the radio. The discovery of a first radio quiet MSP is, however, a caution; the high computational burden for GeV MSP discovery means that the present $\sim 1 \%$ radio quiet fraction is surely an underestimate. Still such MSP are rare.

The new Fermi discoveries also push us to somewhat lower $\dot{E}$ (Guillemot et al. 2016), with a few detections below $10^{33.5} \mathrm{erg} \mathrm{s}^{-1}$. In general the $\gamma$-ray efficiency is higher for the low $\dot{E}$ objects, but there are exceptions. Accurate measurement of the efficiencies requires not only kinematic distances and Shklovskii corrections, but good understanding of the beaming and its evolution with $\dot{E}$. With the increased Pass 8 LAT sensitivity, the measurements of 'under-luminous' PSR can also teach about these beaming corrections, and possibly about fainter high energy emission zones (Romani et al. 2011). Careful analysis of the non-detections remains important for defining the high energy 'death line', which should also help in interpreting the pulsar electrodynamics.

On the particle side, the increased number and quality of Chandra PWN images has refined our picture of the wind flow. The compilations of Pavlov, Kargaltsev \& colleagues (e.g. Kargaltsev et al. 2017), display a variety of morphologies, but emphasize the prevalence of the underlying pattern of an equatorial torus and polar jets. We can hope that as understanding of the pulsar electrodynamics improves we can connect the detailed morphologies to the pulsar spin geometry (Lyubarsky \& Kirk 2001; Porth et al. 2015). In my view the connection with the viewing angle $\zeta$ is already robust, but the morphological dependence on magnetic inclination $\alpha$ is not yet secure. One particular puzzle is the long, swept-back, but very narrow jets seen in several well resolved PWNe, eg. Geminga (Posselt et al. 2017). The narrowness of these jets indicates continuous collimation and the very hard X-ray spectra indicates either rapid transport along the jets or re-acceleration at large distance. It will be interesting to see if MHD models can explain these phenomena.

Many PWNe jet/counter jet systems appear asymmetric. In most cases this can be ascribed to either Doppler boosting (if $\vec{\Omega}$ is well out of the plane of the sky) or ram pressure confinement. However in at least one example, MSP J2124-3358, evidence from 
the enclosing bow shock suggests that the momentum flux is truly asymmetric with one hemisphere producing up to $10 \times$ the flux of the other (Romani, Slane \& Green 2017). In simple electrodynamics, offset dipoles can produce Harrison-Tademaru type momentum asymmetries (Lai, Chernoff \& Cordes 2001), but these are only of order unity. If we find more examples of large intrinsic asymmetry, this will be an important challenge to MHD PWN models.

It has become increasingly interesting to think about how the PWN $e^{ \pm}$energy escapes to the ISM. On large scales we have some information from resolved ICS spectral evolution (e.g. Van Etten \& Romani 2012), and HESS and, increasingly HAWC (Abeysekara et al. 2017) images can be used to map the energy deposition of nearby energetic pulsars. But a number of striking puzzles remain. The long, misaligned wisps (see Reynolds et al. 2017) extending from the apices of several PWNe seem to be pre-existing ISM structures energized by the pulsar wind, so spectral variation along these structures might reveal the PWN particle distribution. On a smaller scale, the recently discovered UV bow shocks (Rangelov et al. 2016; Rangelov et al. 2017) provide evidence that non-thermal pulsar $e^{ \pm}$can cross the contact continuity to the shocked ISM (Romani, Slane \& Green 2017). A more complete study of these emissions from beyond the PWN contact discontinuity should give us a better picture of the spindown deposition and, perhaps, the origin of the cosmic ray $e^{+}$excess.

Two final ways of probing the pulsar wind look particularly promising to me. For a handful of PWNe, the presence of Balmer emission from the forward shock lets us use atomic lines to probe the bow shock velocity structure (and hence the pulsar momentum deposition). With both the forward ISM shock (Balmer lines, UV continuum) and the reverse PWN shock (synchrotron continuum) observed, these special objects offer good prospects for dissecting the PWN energetics (Romani, Slane \& Green 2017).

Finally, close interacting MSP binaries, especially the so-called black widows with fewhour orbital periods and $R_{\odot}$-scale separations place a visible object in the pulsar wind at radii much smaller than the typical $10^{3}-10^{4} \mathrm{AU}$ radius of isolated $\mathrm{PWN}$ termination shocks. If we can understand the dramatic heating of the black widow companions, this offers a new window into the spindown energetics at a new characteristic separation from the pulsar. Certainly photon heating is important and as noted above, this is dominated by the GeV flux for these energetic MSP. In several cases the required heating is well above the flux that we observe, but we should not forget that the companions of these (likely spin-aligned) MSP subtend a zone even more equatorial than the Earth line-ofsight. Careful bolometric measurement of GeV-heated companions thus offers a second cut at the $\gamma$-ray beam's distribution on the sky. Further in at least a few cases, the companion heating seems dominated by a small portion of the surface. This suggests that the PWN particles themselves can be ducted to the companion surface (Sanchez \& Romani 2017). This too offers an energy-integrating probe of the pulsar spindown output, now drawn from the particle sector.

\section{Prospects}

The Fermi LAT sky survey has done a lot for us by providing an essentially unbiased sample of nearby energetic pulsars. But the quest to use this finding list to probe the fundamentals of pulsar output is just beginning. It will take some time to collect the precision parallaxes and multi-wavelength (esp. X-ray and optical) studies of the PWN outflow and its impact on the ISM and binary companions required to map out the wind powers and angular distributions. The reward will be measurement of the strong latitudinal (and possible hemispheric) asymmetries in the pulsar wind. If we can trace patterns 
in these asymmetries to the angles $\alpha$ and $\zeta$ we can connect with the underlying pulsar machine. Such patterns present important targets for magnetosphere/wind simulations.

This work was supported in part by NASA grants G06-17059X and NNM17AA26C.

\section{References}

Abeysekara, A. U., Albert, A., \& Alfaro, R., et al. 2017, ApJ, 834, 40 Abdo, A. A., Ajello, M., \& Baldini, L., et al. 2013, ApJS, 208, 17 Guillemot, L., Smith, D. A., \& Laffon, H., et al. 2016, A\&SA, 587, 109 Kalapotharakos, OC, Harding, A. K., Kazanas, D., \& Brambilla, G. 2017, ApJ, 842, 80 Kargaltsev, O., Pavlov, G. G., Klinger, N., \& Rangelov, B. 2017, J. Plasma Phys., submitted Lai, D. Chernoff, D. F., \& Cordes, J. M. 2001, ApJ, 549, 1111

Lyubarsky, Y. \& Kirk, J. G. 2001, ApJ, 547, 437

Philippov, A. A. \& Sptkovsky, A. 2017, ApJ, submitted; arXiv170704323

Pierbattista, M., Harding, A. K., \& Grenier, I. A. et al. 2015, A\& $A, 575,3$

Porth, O., Vorster, M. J., Lyutikov, M., \& Engelbrecht, N. E. 2016, MNRAS, 560, 4135

Posselt, B., Pavlov, G. G., Slane, P. O. et al. 2017, ApJ, 835, 66

Rangelov, B., Pavlov, G. G., \& Kargaltsev, O. et al. 2016, ApJ 831, 129

Rangelov, B., Pavlov, G. G., \& Kargaltsev, O., et al. 2017, ApJ, 835, 264

Reynolds, S. R., Pavlov, G. G., \& Kargaltsev, O., et al. 2017, SSRv, 207, 175

Romani, R. W., Kerr, M., \& Craig, H. A. et al. 2011, ApJ, 738, 1114

Romani, R. W., Slane, P.,\& Green, A. 2017, ApJ, in press

Sanchez, N. \& Romani, R. W. 2017, ApJ, 845, 42

Van Etten, A. \& Romani, R. W. 2012, ApJ, 755, 151

Watters, K. P., Romani, R. W., Weltevrede, P., \& Johnston, S. 2009, ApJ, 695, 1289 